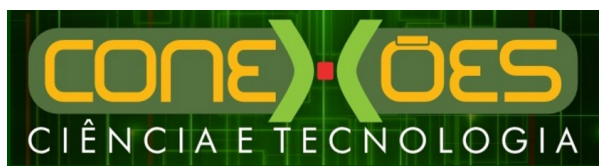

\title{
EFEITO DA EXPECTATIVA COM RELAÇÃO ÀS INFORMAÇÕES SOBRE NÉCTAR E SUCO TROPICAL DE CAJU NA ACEITAÇÃO DO CONSUMIDOR
}

\author{
MARINA CABRAL REBOUÇAS, MARIA FLÁVIA AZEVEDO DA PENHA, \\ CARLOS ELIARDO BARROS CAVALCANTE, ANA CRISTINA DA SILVA MORAIS \\ Instituto Federal de Educação, Ciência e Tecnologia do Ceará (IFCE) \\ <marina_reboucas@hotmail.com>.<flavia.azevedo@ifce.edu.br> \\ <eliardo@ifce.edu.br>.<crisana_2@gmail.com>
}

DOI: $10.21439 /$ conexoes.v11i4.877

\begin{abstract}
Resumo. A expectativa que o consumidor tem sobre um produto pode ser gerada por atributos externos e não sensoriais como informações sobre o produto. Portanto, a rotulagem pode exercer um grande impacto sobre a aceitação dos alimentos. O objetivo deste trabalho foi verificar a percepção do consumidor em relação a diferentes bebidas de frutas atravéis da avaliação da influência das informações na aceitação dos consumidores. Somente o néctar de caju de marca comercial foi utilizado como amostra para a avaliação da expectativa do consumidor em relação às definições de néctar e suco tropical de caju adoçado. $\mathrm{O}$ processo foi realizado em três fases, às cegas, de expectativa e informada, utilizando-se o teste de escala hedônica para avaliar a aceitação dos consumidores em relação às amostras. A expectativa gerada em relação às informações sobre néctar de caju foi confirmada pelas características sensoriais do produto, sem alteração da aceitação. Os resultados individuais mostraram que as informações fornecidas causaram um conflito na percepção do suco e néctar pelo consumidor. Conclui-se que a falta de informação sobre a composição das diferentes bebidas de frutas disponíveis no mercado podem resultar numa percepção equivocada pelo consumidor. Desta forma, medidas que divulguem a legislação relacionada às bebidas de frutas podem ajudar os consumidores a fazer escolhas mais conscientes baseadas nas características reais dos produtos
\end{abstract}

Palavras-chaves: Bebidas de frutas. Estudo com consumidores. Legislação de alimentos. Percepção do consumidor

\begin{abstract}
The consumer expectation about a product can be generated through external and non sensorial attributes, such as the product information. Therefore, the product labelling can have a great impact on the consumer acceptance of food. The aim of this study was to verify the consumer perception in relation to different fruit beverages through the evaluation of information regarding consumers' acceptance. Only the cashew nectar from a commercial trademark was used as sample to evaluate the consumer expectations in relation to nectar definitions and to tropical sweetened cashew juice. The process was carried out in three phases, blind, expectation and informed, using the hedonic scale to evaluate the consumers' acceptance. The expectation created in relation to the information about the cashew nectar was confirmed by the product sensorial characteristics, without any changes. The single results showed that the information given caused a conflict in the consumer perception of the juice and nectar. It is concluded that the lack of information about the composition of different fruit beverages available on the market can result in the consumer mistaken perception. Thus, measures that disseminate the related legislation related to fruit beverages may lead consumers to make the right choices, being conscious of the right characteristics of a product
\end{abstract}

Keywords: Fruit beverage. Study with consumers'. Food legislation. Consumers perception’. 


\section{INTRODUÇÃO}

A inclusão de frutas e seus produtos na dieta é uma recomendação nutricional 61 relacionada à saúde, tendo em vista que estes alimentos fornecem quantidades apreciáveis de 62 vitaminas, minerais e fibras, sendo compostos majoritariamente por água (75 a $95 \%)$ e 63 carboidratos (5 a $25 \%$ ), os quais estão presentes geralmente na forma de sacarose, frutose e 64 glicose(PRADO, 2009).

O consumo de bebidas não alcoólicas vem aumentando no Brasil nos últimos anos. Desde 2005 a participação destes produtos no mercado de bebidas passou de $51,6 \%$ para $53,2 \%$, o que levou a um aumento do consumo per capita de $168,4 \mathrm{~L}$ para $206,7 \mathrm{~L}$ ao ano. Neste mercado, houve um crescimento no consumo de produtos a base de frutas (suco pronto para beber, concentrado e em pó, néctares e refrescos) desde o ano de 2005 até 2010. Nesse contexto, a região Nordeste destaca-se com o maior crescimento no consumo destes produtos em relação às demais regiões do país, crescendo $8,9 \%$ no ano de 2010, 1,4\% acima da média nacional (ABIR 2010).

O crescente mercado brasileiro de suco de fruta tem sido influenciado, principalmente, pelos produtos prontos para beber. Estes devem atender a uma legislação específica, estando de acordo com a definição, classificação, registro, padronização e requisitos de qualidade,atendendo também a legislação específica para rotulagem de alimentos embalados(FERRAREZI; SANTOS; MONTEIRO, 2010).

A legislação brasileira de suco de fruta é bastante abrangente, sendo contemplados diferentes tipos como suco desidratado, integral, reconstituído, concentrado, integral, misto, tropical, néctar e polpa. Por meio da rotulagem dos alimentos, o consumidor pode se orientar sobre a qualidade e a quantidade dos constituintes nutricionais dos produtos, podendo promover escolhas alimentares apropriadas (ARAUJO; ARAUJO 2001; COUTINHO; RECINE, 2007), sendo indispensável,no entanto, a fidedignidade das informações.

No que diz respeito à legislação que define e classifica os diferentes produtos de sucos de frutas, esta possui lacunas que favorecem a interpretação equivocada do consumidor e/ou do produtor, e possibilitam a introdução de sucos não regulamentados no mercado. Este fato é agravado pelas sucessivas alterações nas normas e pela falta de padronização da terminologia empregada, bem como pela ausência de definições essenciais, dificultando a consulta e o conhecimento da legislação (FERRAREZI; SANTOS; MONTEIRO, 2010).

Quando se trata do consumo de um produto ali- mentício a expectativa que o consumidor tem sobre este produto assume um importante papel, pois pode, inclusive, aumentar ou diminuir a intenção de compra deste mesmo antes dele ser experimentado (NORONHA, 2003). São diversos os fatores individuais que podem influenciar a percepção do consumidor a cerca das características sensoriais de um determinado produto (CARDELLO, 1994; NORONHA; DELIZA; SILVA, 2005).

A expectativa que o consumidor tem sobre um produto pode ser gerada por atributos externos e não sensoriais, tais como informações sobre o produto (CAPORALE; MONTELEONE, 2004, CAPORALE et al. 2006; DELIZA; ROSENTHAL; SILVA. 2003) , a embalagem e o rótulo (CARRILLO; VARELA; FISZMAN, 2012; REBOLLAR et al., 2012, RIBEIRO M et al. 2008). Sendo assim, a rotulagem desempenha um importante papel no sentido de capturara atenção do consumidor (ARES; DELIZA 2010), podendo exercer um grande impacto sobre a aceitação dos alimentos (BANOVIĆ et al. 2010; IMM; LEE; LEE, 2012, TORRES-MORENO et al. 2012).

Neste sentido, os fabricantes devem utilizar a embalagem dos alimentos para atrair o consumidor, mas também para gerar expectativas apropriadas com relação ao produto (ARES; DELIZA 2010).

Neste contexto, a fim de se analisar a percepção do consumidor frente a diferentes bebidas de frutas, objetivou-se avaliar a influência das informações referentes à composição do néctar e suco tropical adoçado de caju na aceitação dos consumidores.

\section{MATERIAIS E MÉTODOS}

Para avaliar a influência das informações sobre néctar e suco tropical adoçado na aceitação dos consumidores foi utilizado como amostra néctar de caju de marca comercial. A amostra foi obtida no comércio local de Fortaleza - CE, tendo-se o cuidado de obter produtos provenientes de um mesmo lote. A medida da expectativa do consumidor com relação às definições de néctar e suco tropical adoçado de caju foi avaliada em três fases segundo metodologia proposta por Deliza e MacFie (1996), consistindo em uma avaliação às cegas, de expectativa e informada.

Inicialmente, realizou-se a fase de avaliação às cegas (C) onde as amostras foram servidas em copos descartáveis codificados com números de três dígitos aleatórios. Ao provador foi solicitado que avaliasse o quanto gostou ou desgostou das mesmas de um modo geral utilizando a escala hedônica estruturada mista de 
nove pontos (9 = "gostei muitíssimo"; 5 = "nem gostei, nem desgostei"; 1 = "desgostei muitíssimo").

A fase seguinte, de avaliação da expectativa (E), consistiu em apresentar ao provador informações sobre os dois tipos de produtos. As informações dadas foram adaptações das definições trazidas pela instrução normativa no 12 de 04 de setembro de 2003 (BRASIL. 2003) para o suco tropical adoçado e néctar de frutas. Foi também informado o teor mínimo de polpa de caju exigido para cada tipo de produto de acordo com a legislação (BRASIL, 2003). Desta forma, utilizou-se as seguintes informações para néctar e suco tropical de caju, respectivamente: "Néctar é uma bebida destinada ao consumo direto, obtida da diluição em água potável da parte comestível da fruta e açúcar, podendo ser adicionada de ácidos. No caso do néctar de caju o teor mínimo de polpa exigido pela legislação brasileira é de 15\%"; "Suco tropical adoçado é uma bebida destinada ao consumo direto, obtida pela dissolução, em água potável, da polpa de fruta e açúcar, com cor, aroma e sabor característicos da fruta. No caso do suco tropical adoçado de caju o teor mínimo de polpa exigido pela legislação brasileira é de 25\%.". Aos consumidores foi solicitado que avaliassem as informações e relatassem o quanto acham que gostariam destes produtos utilizando a escala já citada.

A fase de avaliação informada (I) consistiu em servir a amostra juntamente com as informações equivalentes ao produto que iria ser avaliado, destacando-se que estas informações eram sobre o produto que seria degustado. Ao provador foi solicitado que avaliasse o quanto gostou ou desgostou de um modo geral utilizandoa escala hedônica.

É importante ressaltar que nas fases de avaliação as cegas e informada foi utilizada apenas a amostra de néctar de caju para representar os dois produtos (néctar e o suco tropical adoçado de caju), ou seja, nestas duas fases o consumidor provou o mesmo produto.

A análise foi realizada em cabines individuais, sob incidência de iluminação artificial do tipo luz do dia, no Laboratório de Análise Sensorial da Universidade Federal do Ceará. As amostras foram servidas seguindo um delineamento balanceado completo de forma monádica sequencial. Participaram do teste 42 consumidores de sucos de frutas industrializados, sendo a maioria do sexo feminino (64\%), com idade entre 18 e 25 anos $(76 \%)$ e estudantes de graduação $(88 \%)$. A maior parte dos consumidores declarou gostar muitíssimo ou muito de suco de caju (79\%), com uma frequência de consumo de suco de frutas diária ou semanal $(81 \%)$. Os dados referentes às características sócio-demográficas e de consumo dos participantes estão apresentados na Tabela 1

Para avaliar a diferença entre a expectativa do consumidor frente às informações dadas sobre os dois produtos aplicou-se uma ANOVA $(\alpha=0,05)$ entre as médias da fase de expectativa. O teste $t(\alpha=0,05)$ foi utilizado para avaliar a influência da expectativa gerada pelas informações na aceitação das amostras. Para isto, as diferenças entre os escores hedônicos foram calculados para avaliar diferenças significativas entre as amostras pareadas (fase de expectativa $\mathrm{x}$ fase cega, fase informada $x$ fase cega e fase da expectativa $x$ fase informada (LANGE; ROUSSEAU; ISSANCHOU, 1998).

\section{RESULTADOS E DISCUSSÃO}

Houve diferença significativa na expectativa gerada pelas informações sobre suco tropical e néctar de caju Tabela 2. A maior média de aceitação $(7,2)$ foi obtida para a informação sobre suco tropical, o que demonstra que estas causaram uma maior expectativa positiva nos consumidores. A maioria dos consumidores alegou que gostariam do suco tropical de caju devido ao seu elevado teor de polpa, sendo esta possivelmente a razão de sua maior aceitação.

A falta de significância entre a diferença das médias da fase de expectativa e cega $(\mathrm{E}-\mathrm{C})$ e informada $\mathrm{e}$ cega (I - C) para a amostra de néctar de caju indica que houve uma confirmação da expectativa do consumidor Tabela2 Isto mostra que os consumidores sabiam exatamente o quanto iriam gostar deste produto. Os consumidores tinham uma baixa expectativa (média 6,0) com relação a este produto, a qual foi confirmada ao degustálo. Este resultado está em desacordo com a atual percepção que os consumidores brasileiros têm sobre esse produto. Em geral, os consumidores tendem a achar que a bebida tipo néctar é de qualidade superior aos demais sucos prontos para beber.(FERRAREZI; SANTOS; MONTEIRO 2010), argumentam que a expressão "adoçado", que deve estar presente no rótulo do suco e do suco tropical e, ausente no néctar, leva o consumidor a elevar a qualidade deste último, permitindo uma interpretação equivocada. Outra questão trazida por estes autores é que a palavra néctar, como definição trazida nos dicionários, está associada no inconsciente brasileiro a uma bebida dos deuses, à base de mel, deliciosa, saborosa, com propriedade de avivar os sentidos, conservar a eterna juventude e propiciar a imortalidade àqueles que a bebiam, sendo desta forma considerada de qualidade superior. Pela legislação brasileira este termo não tem este mesmo significado, o que poderia justificar a interpretação equivocada do consumidor.A 
Tabela 1: Dados sócio-demográficos e características de consumo dos participantes $(\mathrm{n}=42)$.

\begin{tabular}{ll}
\hline Características & $\%$ de consumidores \\
\hline Sexo & $64 \%$ \\
Feminino & $36 \%$ \\
Masculino & \\
Idade (anos) & $76 \%$ \\
$18-25$ & $21 \%$ \\
$26-35$ & $3 \%$ \\
$36-45$ & \\
Escolaridade & $88 \%$ \\
Superior Incompleto & $12 \%$ \\
Pós - Graduação & \\
Quanto gosta de caju ? & $43 \%$ \\
Muitíssimo & $36 \%$ \\
Muito & $21 \%$ \\
Moderadamente & \\
Frequência de consumo de suco de frutas & $50 \%$ \\
Diariamente & $31 \%$ \\
Semanalmente & $10 \%$ \\
Quinzenalmente & $9 \%$ \\
\hline Mensalemente &
\end{tabular}

falta de conhecimento do consumidor sobre a composição do néctar e as informações trazidas nos rótulos se tornou uma vantagem para o produtor, devido a esta percepção equivocada do consumidor frente aos diferentes produtos no mercado (TURRA et al., 2006). A informação sobre o teor mínimo de polpa de caju trazido neste produto pode ter levado o consumidor a diminuir a expectativa com relação a este. Alguns provadores que atribuíram notas mais baixas justificaram sua avaliação dizendo que o teor de polpa no produto era baixo. Estes resultados demonstram que ao ser devidamente informado sobre as características do produto o consumidor tende a fazer uma avaliação mais real sobre o mesmo.

Tabela 2: Médias do teste de escala hedônica para a fase às cega, de expectativa e informada.

\begin{tabular}{c|c|c}
\hline - & Néctar & Suco \\
\hline Cega & 6,1 & 6,3 \\
Expectativa & $6^{b}$ & $7,2^{a}$ \\
Informada & 6,5 & 6,6 \\
E - C & $0,1^{n s}$ & $0,9^{*}$ \\
I - C & $0,4^{n s}$ & $0,3^{n s}$ \\
I - E & - & - \\
\hline ab Difere significativamente $(\alpha=0,05)$ \\
ns não significativo pelo teste $(\mathrm{p} \geq 0,05)$ \\
* significativo pelo teste $\mathrm{t}(\mathrm{p} \leq 0,05)$
\end{tabular}

As informações dadas sobre o suco tropical de caju causaram uma desconfirmação da expectativa do consumidor. Neste caso houve uma desconfirmação negativa da expectativa, o que indica que o produto em teste cego é pior do que o esperado. Apesar da alta expectativa gerada pelas informações, estas não foram suficientes para elevar a aceitação do produto, o que pode ser observado pela falta de significância entre a diferença de médias da fase informada e cega. Possivelmente, o fato de ter sido utilizado um produto com menor teor de polpa, no caso um néctar, tenha causado esta desconfirmação na expectativa do consumidor.

Quando se trabalha com cálculo de médias em testes afetivos há perda de informações em decorrência da segmentação dos consumidores em função de suas preferências. Deste modo, em testes com número elevado de consumidores frequentemente ocorre uma segmentação dos indivíduos, enquanto que alguns gostam das amostras avaliadas e lhes atribuem notas elevadas, em torno de 7 e 9, outros não as apreciam e dão valores mais baixos, 5 ou menor. Quando se trabalha com a média obtêm-se um valor intermediário que pode não representar nenhum dos dois segmentos. Em virtude deste problema, se torna útil a apresentação dos resultados em gráficos onde cada ponto irá corresponder a um consumidor. As Figuras 1 e 2 trazem representadas no eixo das abscissas as diferenças entre as médias da expectativa e do teste cego $(\mathrm{E}-\mathrm{C})$ e, na ordenada a diferença entre o valor da avaliação informada e do teste 
Tabela 3: Representação dos percentuais de consumidores que se distribuíram nas regiões de assimilação e contraste, os sem definição e os que não apresentaram efeito com relação à apresentação das informações sobre néctar e suco tropical de caju $(n=42)$.

\begin{tabular}{lcc}
\hline Efeitos & \multicolumn{2}{c}{ \% de consumidores } \\
\hline & Néctar & Suco Tropical \\
\hline ASSIMILAÇÃO & & \\
Desconfirmação Negativa & $26 \%$ & $36 \%$ \\
Desconfirmação Positiva & $22 \%$ & $17 \%$ \\
CONTRASTE & & \\
Desconfirmação Negativa & $0 \%$ & $9 \%$ \\
Desconfirmação Positiva & $5 \%$ & $5 \%$ \\
Sem efeito (C = E = I) & $2 \%$ & $7 \%$ \\
Efeitos não claros & $45 \%$ & $26 \%$ \\
\hline
\end{tabular}

cego (I - C). Neste tipo de gráfico muitas vezes os pontos de diferentes consumidores coincidem. Sendo assim, para facilitar a visualização dos efeitos de assimilação e contraste, calculou-se o percentual de indivíduos em cada um dos quatro quadrantes, bem como aqueles onde as médias nas três etapas foram iguais indicando que as informações não surtiram efeito $(\mathrm{C}=\mathrm{E}=\mathrm{I})$ e os que seguiram efeitos não claros 3

As equações de ambos os gráficos mostram uma inclinação positiva, o que mostra que a maioria dos consumidores seguiu o modelo de assimilação das informações dadas. Neste tipo de modelo, os consumidores assimilam a informação dada aproximando a sua aceitação da expectativa que foi criada, seja essa positiva ou negativa (VILLEGAS; CARBONELL; COSTELL, 2008). Em ambos os casos, a assimilação foi positiva,pois houve um aumento da aceitação final do produto.

O baixo coeficiente da regressão $\left(\mathrm{R}^{2}\right)$ obtido por ambas as amostras indicam que a assimilação não foi completa e que outros efeitos ocorreram. O néctar obteve um coeficiente mais baixo $(0,267)$, possivelmente $o$ fato da maioria dos consumidores $(45 \%)$ terem seguido efeitos não claros influenciou na obtenção deste valor. Estes tipos de efeitos ocorrem em duas situações, uma delas é quando a avaliação informada é igual à cega ( $\mathrm{I}=$ C) e a expectativa é maior ou menor que a avaliação às cegas $(\mathrm{E}>\mathrm{C}$ ou $\mathrm{E}<\mathrm{C}$ ), ou então, quando a expectativa é igual a avaliação cega $(\mathrm{E}=\mathrm{C})$ e a informada é maior ou menor que a cega $(\mathrm{I}>\mathrm{C}$ ou $\mathrm{I}<\mathrm{C})$. No primeiro caso, tanto a desconfirmação positiva quanto a negativa parecem ocorrer, no entanto, não afetam a aceitação. Por outro lado, há confirmação da expectativa quando $\mathrm{E}=$ $\mathrm{C}$, porém a diferença entre a avaliação

informada e a cega parece ocorrer de forma aleató-

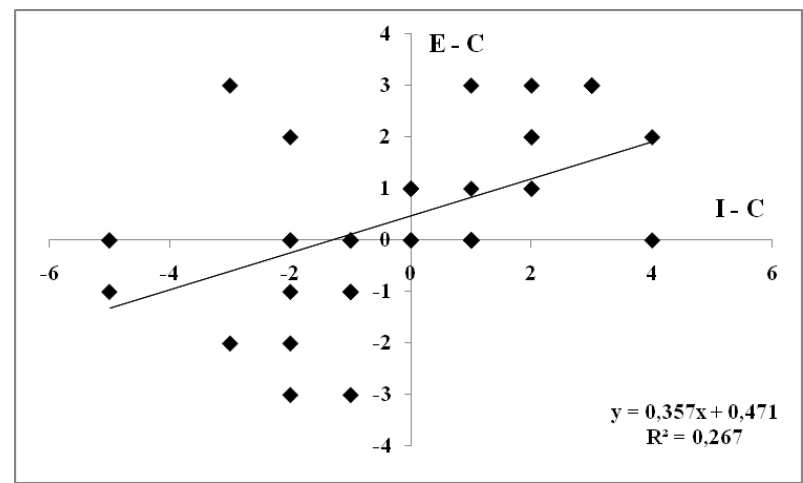

Figura 1: Representação gráfica dos efeitos individuais da expectativa gerada pelas informações sobre néctar de caju no teste de escala hedônica.

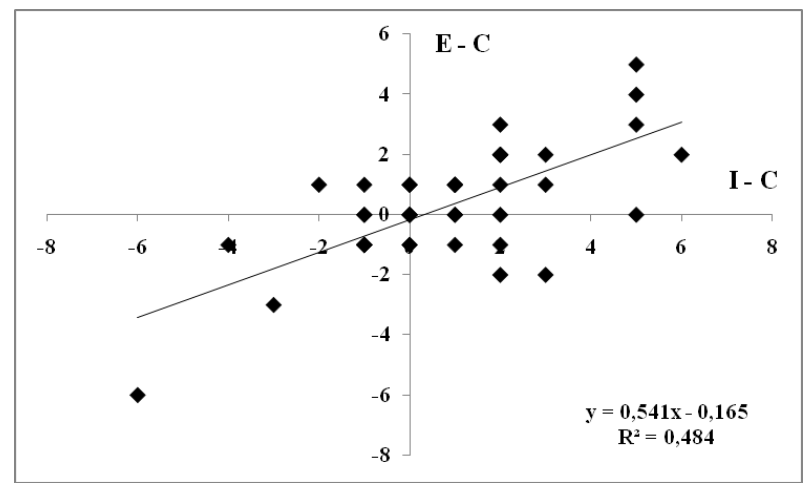

Figura 2: Representação gráfica dos efeitos individuais da expectativa gerada pelas informações sobre suco tropical de caju no teste de escala hedônica. 

DO CONSUMIDOR

ria (BEHRENS; VILLANUEVA; SILVA, 2007). Algumas das hipóteses para estes efeitos, as quais algumas delas também podem explicar o comportamento encontrado nesta pesquisa, são: falta de julgamento crítico e problemas relacionados ao uso da escala hedônica de nove pontos, induzidos pelos efeitos contextuais e numéricos sobre as respostas individuais e a liberdade dos julgadores em expressarem a sua opinião (VILLANUEVA 2003). Outra hipótese pode ser o fato, de como já exposto, as informações dadas com relação à concentração de polpa do néctar terem gerado uma confusão na avaliação do consumidor acostumado a associar este produto a uma qualidade superior. Para o suco tropical, a maioria dos consumidores $(36 \%)$ seguiu o modelo de assimilação com desconfirmação negativa da expectativa. Isto demonstra que os consumidores assimilaram a alta expectativa gerada pelas informações sobre a composição do suco aumentando a aceitação final do produto. Neste caso, a assimilação também não foi completa $\left(R^{2}=0,484\right)$, no entanto mais elevada do que a obtida pelo néctar.

\section{CONCLUSÃO}

As informações sobre o suco tropical adoçado de caju geraram uma maior expectativa positiva do que as do néctar. Ao se avaliar as respostas individuais, a maioria dos consumidores assimilou a alta expectativa gerada com relação às informações sobre o suco tropical de caju aumentando a sua aceitação final.

A expectativa gerada com relação às informações sobre néctar de caju foi confirmada pelas características sensoriais do produto não modificando a aceitação dos consumidores frente a este produto. Com relação aos resultados individuais, estes mostraram que as informações fornecidas causaram um conflito na percepção do consumidor a cerca deste produto, onde a grande maioria seguiu efeitos não claros de expectativa.

Por meio deste trabalho podemos constatar que a falta de informação sobre a composição das diferentes bebidas de frutas disponíveis no mercado fazem com que o consumidor tenha uma percepção equivocada sobre os mesmos. Desta forma, medidas que divulguem a legislação com relação às bebidas de frutas podem levar os consumidores a fazerem escolhas mais conscientes baseados nas características reais dos produtos.

\section{REFERÊNCIAS}

ABIR. Associação Brasileira das Indústrias de Refrigerantes e Bebidas Não-alcoólicas. Dados de mercado 2011. 2010. Disponível em: <http: //abir.org.br/2011/10/24/dados-de-mercado-2011/>. Acesso em: 25/01/2015.

ARAUJO, A. C. M. F.; ARAUJO, W. M. C. Adequaçäo à legislaçäo vigente, da rotulagem de alimentos para fins especiais dos grupos alimentos para dietas com restriçäo de carboidratos e alimentos para dieta de ingestäo controlada de açúcares. 2001.

ARES, G.; DELIZA, R. Identifying important package features of milk desserts using free listing and word association. Elsevier, 2010.

BANOVIĆ, M.; GRUNERT, K. G.; BARREIRA, M. M.; FONTES, M. A. Consumers quality perception of national branded, national store branded, and imported store branded beef. Elsevier, 2010.

BEHRENS, J. H.; VILLANUEVA, N. D.; SILVA, M. A. Effect of nutrition and health claims on the acceptability of soyamilk beverages. International journal of food science \& technology, Wiley Online Library, 2007.

BRASIL, R. F. D. Ministério da agricultura, pecuária e abastecimento. aprova a instrução normativa sobre padrões de identidade para sucos tropicais e néctares. Diário Oficial [da] República Federativa do Brasil, 2003.

CAPORALE, G.; MONTELEONE, E. Influence of information about manufacturing process on beer acceptability. 2004.

CAPORALE, G.; POLICASTRO, S.; CARLUCCI, A.; MONTELEONE, E. Consumer expectations for sensory properties in virgin olive oils. 2006.

CARDELLO, A. V. Measurement of food preferences. : Springer, Boston, MA, 1994. ISBN 978-1-4615-2171-6.

CARRILLO, E.; VARELA, P.; FISZMAN, S. Effects of food package information and sensory characteristics on the perception of healthiness and the acceptability of enriched biscuits. 2012.

COUTINHO, J. G.; RECINE, E. Experiências internacionais de regulamentação das alegações de saúde em rótulos de alimentos. 2007.

DELIZA, R.; MACFIE, H. J. The generation of sensory expectation by external cues and its effect on sensory perception and hedonic ratings: a review. 1996. 

DO CONSUMIDOR

DELIZA, R.; ROSENTHAL, A.; SILVA, A. L. S. Consumer attitude towards information on non conventional technology. 2003.

FERRAREZI, A. C.; SANTOS, K. O.; MONTEIRO, M. Avaliação crítica da legislação brasileira de sucos de fruta, com ênfase no suco de fruta pronto para beber. 2010 .

IMM, B.; LEE, J. h.; LEE, S. H. Effects of sensory labels on taste acceptance of commercial food products. Elsevier, 2012.

LANGE, C.; ROUSSEAU, F.; ISSANCHOU, S. Expectation, liking and purchase behaviour under economical constraint. Elsevier, 1998.

NORONHA, R. L. F. A expectativa do consumidor e sua influencia na aceitação e percepção sensorial de cafe soluvel. Tese (Programa de Pós-Graduação em Tecnologia de Alimentos,) — Universidade Estadual de Campinas, Faculdade de Engenharia de Alimentos, Campinas, SP, 2003.

NORONHA, R. L. F.; DELIZA, R.; SILVA, M. A. A. P. A expectativa do consumidor e seus efeitos na avaliação sensorial e aceitação de produtos alimentícios. 2005.

PRADO, A. Mestrado em Ciência e Tecnologia de Alimentos, Composição fenólica e atividade antioxidante de frutas tropicais. 2009.

REBOLLAR, R.; LIDÓN, I.; SERRANO, A.; MARTÍN, J.; FERNÁNDEZ, M. J. Influence of chewing gum packaging design on consumer expectation and willingness to buy. an analysis of functional, sensory and experience attributes. Elsevier, 2012.

RIBEIRO M, M.; DELLA LUCIA, S. M.; BARBOSA, P. B. F.; GALVãO, H. L.; MINIM, V. P. R. Influência da embalagem na aceitação de diferentes marcas comerciais de cerveja tipo pilsen. SciELO Brasil, 2008.

TORRES-MORENO, M.; TARREGA, A.;

TORRESCASANA, E.; BLANCH, C. Influence of label information on dark chocolate acceptability. Elsevier, 2012.

TURRA, C.; FERNANDES, E.; TAGLIAFERRO, F.; BACCHI, M. Assimetria informacional no mercado brasileiro de sucos de laranja. In: Anais do Congresso Brasileiro de Fruticultura. 2006.
VILLANUEVA, N. D. M. Avaliação do desempenho de quatro metodos de escalonamento em testes sensoriais de aceitação utilizando modelos normais aditivos de analise da variancia e mapas internos de preferencia. Tese (Doutorado em Ciência dos Alimentos e Nutrição) - Universidade Estadual de Campinas - Campinas, SP, 2003.

VILLEGAS, B.; CARBONELL, I.; COSTELL, E. Effects of product information and consumer attitudes on responses to milk and soybean vanilla beverages. Wiley Online Library, 2008. 